\title{
Atributos de qualidade da carne de paca (Agouti paca): perfil sensorial e força de cisalhamento
}

[Quality attributes of paca meat (Agouti paca): sensory profile and shear force]

\author{
C. Gomes ${ }^{1}$, L.B. Karam $^{2}$, R.E.F. Macedo ${ }^{2 *}$
}

${ }^{1}$ Aluno de pós-graduação - Escola de Ciências Agrárias e Medicina Veterinária - PUCPR - São José dos Pinhais, PR
${ }^{2}$ Escola de Ciências Agrárias e Medicina Veterinária - PUCPR - São José dos Pinhais, PR

\section{RESUMO}

Avaliaram-se as características sensoriais e determinou-se a força de cisalhamento de cortes de carne de paca (Agouti paca). As análises foram realizadas nos cortes desossados de paleta, lombo e pernil de nove pacas, preparados por cocção até a temperatura interna de $70^{\circ} \mathrm{C}$. A avaliação de aspecto, cor, sabor, odor e maciez foi realizada pela aplicação de teste afetivo a 146 provadores, utilizando-se escala hedônica, e a força de cisalhamento foi determinada pela técnica Warner Bratzler. Na avaliação sensorial, os cortes de paleta, lombo e pernil de paca mostraram diferença significativa $(\mathrm{p}<0,05)$ para os atributos de aspecto e cor. A paleta obteve pontuação mais baixa para o aspecto e mostrou coloração mais forte em relação aos demais cortes. Para a força de cisalhamento, não houve diferença $(p>0,05)$ entre os cortes, que se mostraram igualmente macios. A carne de paca apresentou-se sensorialmente semelhante à carne suína e com boa aceitação pelos consumidores. O estudo evidenciou o potencial da paca como uma espécie silvestre para a produção comercial de carne para o mercado de carnes vermelhas ou exóticas.

Palavras-chave: animais silvestres, aceitação sensorial, maciez

\begin{abstract}
Sensory characteristics and shear force of paca meat (Agouti paca) were assessed in this study. Analyses were performed in the boneless shoulder, loin and ham obtained from nine paca carcasses prepared by cooking until reaching the internal temperature of $70^{\circ} \mathrm{C}$. The evaluation of flavor, aroma, color, appearance and tenderness was carried out by the application of an affective test using the hedonic scale and a 146 consumer panel. Shear force was determined by the Warner-Bratzler technique. Shoulder, loin and ham had significant differences $(p<0.05)$ in appearance and color. Shoulder presented the lowest score for appearance and showed darker color compared to the other cuts. Shear force values had no significant differences $(p>0.05)$ among the cuts, which were similarly tender. Paca meat was found to resemble pork meat in sensory evaluation and had good acceptance by consumers. This study showed the potential of paca (Agouti paca) as a wild species for meat production in the red or exotic meat market.
\end{abstract}

Keywords: game meat, sensory profile; shear force

\section{INTRODUÇÃO}

Para consolidação de consumo de determinada espécie de carne, a qualidade é um fator prioritário e deve atender às exigências e ao perfil do mercado consumidor ao qual se destina (Gularte et al., 2000). O grau de satisfação, derivado do consumo da carne, depende de respostas psicológicas e sensoriais inerentes a cada indivíduo. Os fatores que influenciam na reação de gostar ou não de determinada porção de carne são aparência, maciez, suculência e sabor. As características da carne que contribuem para sua atratividade e palatabilidade são aquelas agradáveis aos olhos, nariz e paladar, dentre as quais sobressaem os aspectos relacionados ao

Recebido em 12 de outubro de 2011

Aceito em 8 de outubro de 2012

*Autor para correspondência (corresponding author)

E-mail: renata.macedo@pucpr.br 
sabor e à suculência (Madruga et al., 2005). Para evocar, medir, analisar e interpretar como as características da carne são percebidas pelos sentidos da visão, olfato, paladar e tato, utiliza-se a análise sensorial (ABNT, 1993). Os testes sensoriais afetivos determinam a aceitação de um produto por parte dos consumidores (Della Torre et al., 2003).

Entre os métodos sensoriais disponíveis para medir a aceitação e a preferência dos consumidores por determinados tipos de alimentos, a escala hedônica estruturada de nove pontos é o método afetivo mais utilizado devido à confiabilidade e validade de seus resultados, bem como à sua simplicidade em ser utilizada pelos provadores. As escalas hedônicas dão a grandeza (intensidade da sensação) e a direção das diferenças entre as amostras. Por meio desse método, é possível descobrir o quanto as amostras diferem entre si e qual a amostra que apresenta maior intensidade do atributo sensorial que está sendo medido (Behrens et al., 1999; Dutcosky, 2011).

De todos os atributos da qualidade sensorial da carne, a maciez é considerada o mais importante pela média de consumidores e parece ser mais valorizada que o odor, o sabor e a cor (Lachowicz et al., 1998; Jeleníková et al., 2008; Salvá et al., 2009). A avaliação da maciez da carne pode ser realizada por métodos subjetivos, como a análise sensorial ou métodos instrumentais, como a medida da força de cisalhamento (Szczesniak, 2002; Lachowicz et al., 1998).

Apesar da tendência de consumo de carne de espécies silvestres observada especialmente em países da Europa, África e América (Hoffman e Wiklund, 2006; Saadoun e Cabrera, 2008) e da potencialidade do mercado de carne de paca (Agouti paca) no Brasil, são inexistentes nas literaturas nacional e internacional consultadas estudos sobre os atributos de qualidade e aceitabilidade da carne dessa espécie silvestre. Nesse contexto, o presente trabalho teve como objetivo a obtenção de dados sobre as características sensoriais e a força de cisalhamento de cortes cárneos de paca.

\section{MATERIAL E MÉTODOS}

Foram utilizadas nove pacas, cinco fêmeas e quatro machos, com idades entre quatro e 11 meses, procedentes do criatório comercial de pacas da Fazenda Experimental Gralha Azul da PUCPR. Este estudo foi aprovado pelo Comitê de Ética no Uso de Animais da PUCPR, com o número de protocolo 342. Os animais foram criados em sistema de confinamento e receberam dieta fornecida ad libitum constituída por frutas maçã e banana - e concentrado especial para cobaias (Nuvital ${ }^{\circledR}$ ) contendo $22 \%$ de proteína bruta e $4 \%$ de extrato etéreo.

Os animais foram abatidos com peso vivo entre 5,03 a $7,35 \mathrm{~kg}$ em matadouro frigorífico habilitado pelo Ibama para animais silvestres e com SIF, localizado na região metropolitana de Curitiba-PR. Previamente ao abate, os animais foram submetidos a jejum e dieta hídrica por um período de oito horas e descanso de duas horas após a chegada ao abatedouro. Após o abate, as carcaças foram refrigeradas em câmara fria até a temperatura de $4^{\circ} \mathrm{C}$ e posteriormente dividas em meias-carcaças e separadas em quatro grandes cortes técnicos: paleta, costela/barriga, pescoço/carré e pernil. Os cortes de paleta, carré (lombo) e pernil foram desossados e utilizados para as análises realizadas.

A avaliação sensorial foi realizada mediante teste afetivo com aplicação de escala hedônica mista, sendo utilizadas as escalas numérica e verbal para se demarcar cada categoria a ser avaliada (Dutcoski, 2011). As amostras foram avaliadas para atributos de afetividade e de intensidade. Os atributos de afetividade avaliados foram aspecto e sabor, enquanto os atributos de intensidade foram odor, cor e maciez. Na atribuição das notas, os provadores utilizaram escala de nove pontos, na qual os atributos de aspecto, com escala variando de ruim a bom, e cor, com escala variando de fraca a forte, foram avaliados visualmente. Os atributos maciez - mole a firme - e sabor - ruim a bom - foram avaliados pela degustação das amostras, e o odor - fraco a forte - foi avaliado pelo olfato. As notas foram atribuídas de acordo com a percepção e a intensidade de cada atributo, sendo a nota 1 correspondente a ruim, mole ou fraco, a nota 5 correspondente a intermediário, e a nota 9 correspondente a bom, firme ou forte para os atributos de aspecto/sabor, maciez e cor/odor, 
respectivamente. $\mathrm{O}$ grupo de provadores foi composto por 146 indivíduos adultos, não treinados, com idades de 18 a 50 anos e que relataram apreciar e consumir carne com regularidade.

O preparo das amostras foi realizado pelo cozimento dos cortes de paleta, lombo e pernil acondicionados em embalagens plásticas termorresistentes em banho-maria até ser atingida a temperatura interna de $70^{\circ} \mathrm{C}$ (AMSA, 1995). Após o cozimento, as amostras foram cortadas em formato cúbico de aproximadamente $1 \mathrm{~cm}^{3}$ e apresentadas aos provadores, mantendose aquecidas. Os provadores receberam as amostras codificadas com três dígitos e desconheciam tanto o corte quanto a espécie animal da qual as amostras eram provenientes. A avaliação dos atributos foi realizada pelo preenchimento de ficha própria de análise.

A força de cisalhamento foi determinada nos cortes de paleta, lombo e pernil previamente cozidos conforme procedimento descrito anteriormente. Após cozimento, seis porções de cada corte foram retiradas em formato cilíndrico de $1,27 \mathrm{~cm}$ de diâmetro e cisalhadas perpendicularmente à orientação das fibras musculares em texturômetro TA-XT2i Texture Analyser (Stable Micro Systems, Surrey, UK), equipado com lâmina Warner-Bratzler, com velocidade de descida da lâmina padronizada em $1,5 \mathrm{~mm} / \mathrm{s}$ a partir da distância de $30 \mathrm{~mm}$ da base do texturômetro (AMSA, 1995; Pires et al., 2002).

Os dados obtidos para os cortes na avaliação sensorial e na determinação da força de cisalhamento foram submetidos à análise de variância, aplicando-se o teste Tukey para a comparação de médias e adotando-se nível de significância de 5\%. Para comparação dos resultados de força de cisalhamento dos cortes de machos e de fêmeas, usou-se o teste $\mathrm{t}$ com significância de $5 \%$. Todos os cálculos foram realizados utilizando-se o software estatístico GraphPad Prism version 3.00 for Windows, San Diego - Califórnia, EUA, 2003.

\section{RESULTADOS E DISCUSSÃO}

$\mathrm{Na}$ avaliação sensorial dos cortes de paca, foi observada diferença $(\mathrm{P}<0,05)$ somente para os atributos de cor e aspecto (Tab. 1).

Tabela 1. Avaliação sensorial dos cortes de paleta, lombo e pernil de paca (Agouti paca)

\begin{tabular}{lccccc}
\hline Corte cárneo & Cor $*$ & Aspecto * & Sabor * & Odor * & Maciez * \\
\hline Paleta & $6,43 \mathrm{a} \pm 1,87$ & $6,01 \mathrm{a} \pm 1,98$ & $6,34 \mathrm{a} \pm 1,82$ & $5,92 \mathrm{a} \pm 2,08$ & $5,92 \mathrm{a} \pm 2,40$ \\
Lombo & $5,57 \mathrm{~b} \pm 2,03$ & $6,62 \mathrm{~b} \pm 1,64$ & $6,46 \mathrm{a} \pm 1,78$ & $5,66 \mathrm{a} \pm 2,07$ & $5,97 \mathrm{a} \pm 2,24$ \\
Pernil & $5,77 \mathrm{~b} \pm 1,95$ & $6,42 \mathrm{ab} \pm 1,66$ & $6,77 \mathrm{a} \pm 1,64$ & $5,99 \mathrm{a} \pm 1,89$ & $5,98 \mathrm{a} \pm 2,20$ \\
\hline
\end{tabular}

*Média \pm desvio-padrão. Médias seguidas de letras diferentes na mesma coluna indicam diferença significativa entre si $(\mathrm{p}<0,05)$.

Escala: nota 1 - corresponde a ruim, mole ou fraco; nota 5 - corresponde a intermediário; nota 9 - corresponde a bom, firme ou forte para os atributos de aspecto/sabor, maciez e cor/odor, respectivamente.

Com relação à cor, foi observada diferença significativa entre os cortes de paca, sendo a paleta o corte que obteve a maior nota média, $6,43 \pm 1,87$, em relação ao pernil e ao lombo $(\mathrm{P}<0,05)$, correspondendo à cor mais forte na escala sensorial utilizada. Nessa escala, cor mais forte correspondia à tonalidade vermelha/marrom mais escura percebida pelos provadores. Dentre os requisitos sensoriais, a cor é o fator determinante nas decisões de compra pelo consumidor (Renerre, 1990; Balog et al., 2008), sendo considerada como o primeiro critério de atratividade da carne. Mais do que qualquer outro fator de qualidade, quando os consumidores não têm acesso ao odor da carne, eles utilizam inicialmente a coloração como indicador de frescor (Tejeda et al., 2008). A cor não afeta a palatabilidade da carne, porém é de grande importância para a decisão de compra pelos consumidores (Brondani et al., 2006). 
$\mathrm{Na}$ avaliação da carne de paca, a paleta, considerada de cor vermelha/marrom mais escura, teve o aspecto menos apreciado pelos provadores, apresentando diferença em relação ao aspecto do lombo $(\mathrm{P}<0,05)$. Contudo, embora a paleta tenha recebido nota mais baixa, seu aspecto foi classificado como intermediário a bom na escala sensorial utilizada. O lombo recebeu a maior nota $(6,62 \pm 1,64)$ para o aspecto e apresentou cor vermelha/marrom menos intensa (menor nota) em relação ao pernil e à paleta. A cor da carne é um dos principais atributos que influencia sua atratividade e, dessa forma, afeta a percepção de seu aspecto pelos consumidores (Lawrie, 2005).

Diferenças físicas e sensoriais entre as espécies silvestres podem ser utilizadas como uma ferramenta de marketing para a carne desses animais, uma vez que diferentes espécies apresentam sabores peculiares em sua carne (Hoffman et al., 2009). Embora a carne de caça proveniente de animais silvestres apresente sabor variável de acordo com cada espécie, os atributos para a avaliação são os mesmos utilizados para as carnes tradicionais, como cor, textura, sabor e suculência (Wiklund et al., 2003). Segundo Hoffman e Wiklund (2006), os consumidores de carne de caça desejam as mesmas características de qualidade encontradas nas carnes de espécies tradicionais, contudo sua expectativa é a de que carnes de caça apresentem atributos sensoriais inferiores aos das carnes tradicionais, particularmente no que se refere à cor, suculência e maciez.

Daszkiewicz et al. (2009) relatam que as carnes de caça normalmente apresentam cor vermelha ou marrom mais escura do que as carnes tradicionais. Essa característica pode levar à falsa percepção de que as carnes de caça apresentam defeitos de qualidade relacionados à condição de estresse pré-abate dos animais. Na realidade, a cor vermelha ou marrom mais escura das carnes de caça é reflexo do maior teor de mioglobina muscular resultante da maior atividade motora e menor eficiência de sangria desses animais quando comparados aos animais tradicionais de açougue.

Para sabor e odor, não foi observada diferença significativa $(p>0,05)$ entre os diferentes cortes avaliados. Em carnes oriundas de espécies animais semelhantes, as diferenças de sabor podem ser atribuídas, entre outros fatores, à dieta fornecida aos animais (Melton, 1990; Rodbotten et al., 2004). Hoffman et al. (2007) observaram alterações no sabor da carne de animais silvestres decorrentes de diferenças na composição da gordura encontrada nos cortes cárneos.

São poucos os estudos relacionados à avaliação sensorial de carne de animais silvestres. Em estudo sensorial com carne de rena (Rangifer tarandus tarandus L.) na Suécia, Wiklund et al. (2003) observaram diferenças $(\mathrm{p}<0,05)$ quanto ao sabor da carne de animais alimentados exclusivamente com pastagem nativa e dos alimentados à base de concentrados. $\mathrm{O}$ estudo mostrou que a carne das renas submetidas à dieta com concentrados apresentou maior intensidade de sabor, descrito como adocicado e semelhante a fígado, e menor intensidade de sabores, descritos como ferroso, ácido, metálico, semelhante a sangue ou à carne ovina. As notas atribuídas à maciez do lombo de renas variaram de 67,8 a 68,0 em uma escala de 100 pontos. $O$ lombo das pacas recebeu pontuação para maciez em proporção semelhante, com nota média de 5,97 em uma escala de 9,0 pontos.

Segundo Hoffman et al. (2007), amostras de lombo de cabras-de-leque (Antidorcas marsupialis), uma pequena gazela africana, foram avaliadas sensorialmente por uma equipe de provadores treinados e receberam notas para os atributos aroma e sabor característicos de carne de caça que variaram de 4,46 a 5,49 em uma escala de 8,0 pontos, na qual o valor 8,0 correspondia à melhor pontuação. Hoffman et al. (2009) também compararam sensorialmente lombo de kudu (Tragelaphus strepsiceros), uma espécie de antílope africano, com o de impala (Aepyceros melampus) e verificaram maiores notas de sabor para a carne de impala e de suculência para a carne de kudu. Para os demais atributos avaliados, aroma, maciez e sabor residual, não houve diferença significativa entre a carne das duas espécies silvestres.

Considerando-se os resultados gerais obtidos na avaliação sensorial, verificou-se boa aceitação pelos provadores da carne de paca com notas próximas a 6,0 (Tab. 1). Na percepção geral dos atributos sensoriais, os provadores relataram a grande semelhança da carne de paca com a carne suína, podendo ser facilmente confundida com esta última. 
$\mathrm{Na}$ avaliação da força de cisalhamento, não foi verificada diferença significativa $(p>0,05)$ entre os cortes avaliados (Tab. 2).

Gularte et al. (2000) observaram correlação positiva entre as notas atribuídas pelos julgadores na avaliação sensorial para maciez da carne e os valores de força de cisalhamento, constatando que menor maciez correspondia à maior força de cisalhamento (Hoffman et al., 2007). A determinação da força de cisalhamento pela técnica de Warner-Bratzler é a metodologia instrumental de avaliação de maciez da carne que melhor se correlaciona com a avaliação sensorial (Tornberg, 1996; Hoffman et al., 2009). Embora os cortes de paca tenham obtido valores numéricos diferentes para a força de cisalhamento e para a maciez, na avaliação estatística, eles não mostraram diferença $(\mathrm{p}>0,05)$ para esse atributo medido tanto pelo método instrumental quanto pelo sensorial.

Tabela 2. Força de cisalhamento obtida nos cortes paleta, lombo e pernil de paca (Agouti paca)

\begin{tabular}{cccc}
\hline Corte cárneo & \multicolumn{3}{c}{ Força de cisalhamento $(\mathrm{kg}) *$} \\
\cline { 2 - 4 } & Machos & Fêmeas & Valor médio do corte \\
\hline Paleta & $2,08 \mathrm{a} \pm 0,55$ & $2,95 \mathrm{a} \pm 0,51$ & $2,56 \mathrm{~A} \pm 0,67$ \\
Lombo & $2,98 \mathrm{a} \pm 0,28$ & $3,94 \mathrm{a} \pm 0,87$ & $3,51 \mathrm{~A} \pm 0,81$ \\
Pernil & $2,63 \mathrm{a} \pm 1,26$ & $2,55 \mathrm{a} \pm 1,09$ & $2,59 \mathrm{~A} \pm 1,09$ \\
\hline
\end{tabular}

*Média \pm desvio-padrão. Médias seguidas de mesma letra minúscula na mesma linha não diferem estatisticamente entre si $(\mathrm{P}>0,05)$. Médias seguidas de mesma letra maiúscula na mesma coluna não diferem estatisticamente entre si $(\mathrm{P}>0,05)$.

$\mathrm{Na}$ literatura internacional são ainda mais escassas as publicações relativas à força de cisalhamento de carne de animais silvestres. Pratiwi et al. (2007) obtiveram valores de 4,66 e $6,65 \mathrm{~kg}$ para a força de cisalhamento do lombo de cabras-selvagens abatidas na Austrália com peso vivo de 5 a $10 \mathrm{~kg}$, respectivamente. Esses valores foram superiores aos obtidos para o lombo das pacas, que foram abatidas com peso vivo médio de $6,2 \mathrm{~kg}$.

Embora alguns trabalhos tenham encontrado diferença entre a força de cisalhamento da carne de fêmeas e machos de animais silvestres (Saadoun e Cabrera, 2008; Daszkiewicz et al., 2009), o sexo dos animais não mostrou influência na força de cisalhamento dos cortes de paca $(\mathrm{P}>0,05)$. Na comparação dos valores de força de cisalhamento da carne de paca com a de capivara, outro roedor comumente encontrado no Brasil, a carne de paca mostrou-se mais macia, com valores para fêmeas de $3,14 \mathrm{~kg}$ e de $2,56 \mathrm{~kg}$ para machos. Os valores relatados por Saadoun e Cabrera (2008) para força de cisalhamento de carne de fêmeas e machos de capivara foram de 5,21 e $5,18 \mathrm{~kg}$, respectivamente.

Mostert e Hoffman (2007) obtiveram valores de 14,27 e $13,92 \mathrm{~kg}$, para a força de cisalhamento de lombo de fêmeas e machos de kudu (Tragelaphus strepsiceros). Por outro lado, Daszkiewicz et al. (2009) observaram valores menores que os encontrados neste estudo para a força de cisalhamento de lombo de machos e fêmeas de veado-vermelho capturados na Polônia. Esses autores relataram valores de força de cisalhamento de 2,06 e $2,72 \mathrm{~kg}$ para a carne de fêmeas e de machos, respectivamente.

Os resultados de força de cisalhamento obtidos para a carne de paca podem defini-la como carne macia, visto que estiveram entre 2,08 e $3,94 \mathrm{~kg}$. Carnes com valores de força de cisalhamento acima de $5,5 \mathrm{~kg}$ são consideradas sensorialmente duras pelo consumidor (Shackelford et al., 1991; Pratiwi et al., 2007).

Além da espécie, idade do animal e teor de água da carne, outros fatores como a forma e a espessura da lâmina usada para cisalhamento, uniformidade, tamanho e formato da amostra, direção das fibras musculares, presença de tecido conectivo e depósitos de gordura, temperatura da amostra e ainda a velocidade de descida da lâmina podem influenciar nos resultados da força de cisalhamento medida por diferentes laboratórios e dificultar a definição de padrões de maciez da carne (AMSA, 1995; Ramos e Gomide, 2007; Wheeler et al., 2007).

O tempo de exposição das amostras a altas temperaturas de cozimento também pode influenciar a força de cisalhamento (Rodbotten $e t$ al., 2004). Pires et al. (2002) citam que parece 
haver um intervalo crítico de temperatura interna no qual as proteínas miofibrilares da carne são desnaturadas. Segundo esses autores, quanto maior o período de tempo em que a carne é mantida no intervalo de temperatura de 70 a $80^{\circ} \mathrm{C}$, maior será o efeito de endurecimento sobre a actomiosina. Assim, a não observação de diferença significativa na força de cisalhamento entre cortes de carne suína avaliados por Pires et al. (2004) foi atribuída ao fato de as amostras do experimento terem permanecido por rápidos períodos de tempo ( 3 a $5 \mathrm{~min}$ ) em contato com esse intervalo crítico de temperatura, não ocasionando efeito significativo sobre o endurecimento da actomiosina. Essa condição de temperatura também pode ter influenciado os resultados da força de cisalhamento dos cortes de paca, pois, por serem cortes de pequeno peso, não tardaram a atingir a temperatura interna de $70^{\circ} \mathrm{C}$, recomendada para o cozimento das amostras previamente à determinação da força de cisalhamento.

\section{CONCLUSÃO}

Os cortes de carne de paca mostraram perfil sensorial semelhante para os atributos de sabor, odor e maciez. A paleta mostrou cor vermelha/marrom mais escura e aspecto menos apreciado que os demais cortes $(\mathrm{P}<0,05)$. Contudo, o aspecto desse corte foi considerado de classificação intermediário a bom na escala sensorial utilizada. A carne de paca mostrou-se de textura macia e apreciada pelos consumidores, sendo considerada sensorialmente semelhante à carne suína. Desse modo, o estudo comprova o potencial da paca como espécie para a produção de carne, podendo-se constituir uma alternativa às demais carnes vermelhas.

\section{REFERÊNCIAS}

ABNT. ASSOCIAÇÃO BRASILEIRA DE NORMAS TÉCNICAS. NBR 12806: fev. 1993, p.8.

AMSA. AMERICAN MEAT SCIENCE ASSOCIATION. Research guidelines for cookery, sensory evaluation, and instrumental tenderness measurements of fresh meat. Chicago: National Livestock and Meat Board, 1995. p.1-33.

BALOG, A.; MENDES, A.A.; ALMEIDA PAZ, I.C.L. et al. Carne de avestruz: rendimentos de carcaça e aspectos físicos e químicos. Cienc. Tecnol. Alim., v. 28, p. $400-407,2008$
BEHRENS, J.H.; SILVA, M.A.A.P.; WAKELING, I.N. Avaliação da aceitação de vinhos brancos varietais brasileiros através de testes sensoriais afetivos e técnica multivariada de mapa de preferência interno. Cienc. Tecnol. Alim., v.19, p.214-220, 1999.

BRONDANI, I.V.; SAMPAIO, A.A.M.; RESTLE J.R. et al. Composição física da carcaça e aspectos qualitativos da carne de bovinos de diferentes raças alimentados com diferentes níveis de energia. Rev. Bras. Zootec., v.35, p.2034-2042, 2006.

DASZKIEWICZ, T.; JANISZEWSKI, P.; WAJDA, S. Quality characteristics of meat from wild red deer (Cervus elaphus 1.) hinds and stags. J. Muscle Foods, v.20, p.428-448, 2009.

DELLA TORRE, J.C.M; RODAS, M.A.B; TADINI, C.C. Perfil sensorial e aceitação de suco de laranja pasteurizado minimamente processado. Cienc. Tecnol. Alim., v.23, p.105-111, 2003.

DUTCOSKI, S.D. Análise Sensorial de Alimentos. 3.ed. Curitiba: Champagnat, 2011. 426 p.

GULARTE, M.A.; TREPTOW, R.O.; POUEY, J.L.O. et al. Idade e sexo na maciez da carne de ovinos da raça Corriedale. Cienc. Rural, v.30, p.485-488, 2000.

HOFFMAN, L.C.; KROUCAMP, M.; MANELY, M. Meat quality characteristics of springbok (Antidorcas marsupialis). 4: Sensory meat evaluation as influenced by age, gender and production region. Meat Sci., v.76, p.774-778, 2007.

HOFFMAN, L.C.; MOSTERT, A.C.; LAUBSCHER, L.L. Meat quality of kudu (Tragelaphus strepsiceros) and impala (Aepyceros melampus): The effect of gender and age on the fatty acid profile, cholesterol content and sensory characteristics of kudu and impala meat. Meat Sci., v.83, p.737-743, 2009.

HOFFMAN, L.C.; WIKLUND, E. Game and venison - meat for the modern consumer. Meat Sci., v.74, p.197-208, 2006.

JELENÍKOVÁ, J.; PIPEK, P.; STARUCH, L. The influence of ante-mortem treatment on relationship between $\mathrm{pH}$ and tenderness of beef. Meat Sci., v.80, p.870-874, 2008.

LACHOWICZ, K.; GAJOWIECKI, L.; DVORAK, J. et al. Texture and Rheological Properties of Meat from Pigs of Different Halothane Genotypes. J. Sci. Food Agri., v.77, p.373-380, 1998.

LAWRIE, R.A. Ciência da carne. 6.ed. Porto Alegre: Artmed, 2005. 384p.

MADRUGA, M.S.; NARAIN, N.; DUARTE, T.F. et al. Características químicas e sensoriais de cortes Comerciais de caprinos srd e mestiços de Bôer. Cienc. Tecnol. Alim., v.25, p.713-719, 2005. 
MELTON, S. Effects of feeds on flavour of red meat a review. J. Anim. Sci., v.12, p.4421-4435, 1990.

MOSTERT, R.; HOFFMAN, L.C. Effect of gender on the meat quality characteristics and chemical composition of kudu (Tragelaphus strepsiceros), an African antelope species. Meat Sci., v.104, p.565-570, 2007.

PRATIWI, N.M.W.; MURRAY, P.J.; TAYLOR, D.G. Feral goats in Australia: A study on the quality and nutritive value of their meat. Meat Sci., v.75, p.168177, 2007.

PIRES, I.S.C; ROSADO, G.P.; AZEVEDO, R.M.C. et al. Composição centesimal de porções de carne suína submetidas a diferentes tratamentos de congelamento e descongelamento. Rev. Nutrição, v.15, p.163-172, 2002.

RAMOS, M.E.; GOMIDE, L.A.M. Avaliação da qualidade de carnes: fundamentos e tecnologias. Viçosa: editora UFV, 2007. 599 p.

RENERRE, M. Review: factors involved in the discoloration of beef meat. Internat. J. Food Sci. Technol., v.25, p.613-630, 1990.

RODBOTTEN, M.; KUBBEROD, E.; LEA, P. et al. A sensory map of the meat universe. Sensory profile of meat from 15 species. Meat Sci., v.68, p.137-144, 2004.

SAADOUN, A.; CABRERA, M.C. A review of the nutritional content and technological parameters of indigenous sources of meat in South America. Meat Sci., v.80, p.570-581, 2008.
SALVÁ, B.K.; ZUMALACÁRREGUI, J.M; FIGUEIRA, A.C. et al. Nutrient composition and technological quality of meat from alpacas reared in Peru. Meat Sci., v.4, p.450-455, 2009.

SHACKELFORD, S.D.; MORGAN, J.B.; CROSS, H.R. et al. Identification of threshold levels for Warner-Bratzler shear force in beef top loin steaks. $J$. Muscle Food, v.2, p.289-296, 1991.

SZCZESNIAK, A.S. Texture is a sensory property. Food Qual. and Pref., v.13, p.215-225, 2002.

TEJEDA, J.F; PEÑA, R.E; ANDRÉS, A.I. Effect of live weight and sex on physico-chemical and sensorial characteristics of Merino lamb meat. Meat Sci., v.80, p.1061-1067, 2008.

TORNBERG, E. Biophysical aspects of meat tenderness. Meat Sci., v.43, p.175-191, 1996.

WHEELER, T.L; SHACKELFORD, S.D; KOOHMARAIE, M. Beef longissimus slice shear force measurement among steak locations and institutions. J. Anim. Sci., v.85, p.2283-2289, 2007.

WIKLUND, E.; JOHANSSON, L.; MALMFORS, G. Sensory meat quality, ultimate $\mathrm{pH}$ values, blood parameters and carcass characteristics in reindeer (Rangifer tarandus tarandus L.) grazed on natural pastures or fed a commercial feed mixture. Food Qual. and Pref., v.7, p.573-581, 2003. 